\begin{tabular}{|c|c|c|c|}
\hline 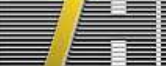 & 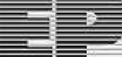 & PuBlisheD & FOR SISSA BY SPRINGER \\
\hline & & & $\begin{array}{l}\text { RECEIVED: April 23, } 2014 \\
\text { ACCEPTED: June 7, } 2014 \\
\text { PUBLISHED: June 23, } 2014\end{array}$ \\
\hline
\end{tabular}

\title{
Rindler/Contracted-CFT correspondence
}

\author{
Reza Fareghbal ${ }^{a, b}$ and Ali Naseh ${ }^{b}$ \\ ${ }^{a}$ Department of Physics, Shahid Beheshti University, \\ G.C., Evin, Tehran 19839, Iran \\ ${ }^{b}$ School of Particles and Accelerators, Institute for Research in Fundamental Sciences (IPM), \\ P.O. Box 19395-5531, Tehran, Iran \\ E-mail: r_fareghbal@sbu.ac.ir, naseh@ipm.ir
}

ABSTRACT: Taking the flat-space limit (zero cosmological constant limit) of the RindlerAdS spacetime yields the Rindler metric. According to the proposal of Flat/contractedCFT correspondence, the flat-space limit on the bulk side of asymptotically AdS spacetimes corresponds to the contraction of the conformal field theory on the boundary. We use this proposal for the Rindler-AdS/CFT correspondence and propose a dual theory for the Rindler spacetime, which is a contracted conformal field theory (CCFT). We show that the two-dimensional CCFT symmetries exactly predict the same two-point functions that one may find by taking the flat-space limit of three-dimensional Rindler-AdS holographic results. Using the Flat/CCFT proposal, we also calculate the three-dimensional Rindler energy-momentum tensor. Since the near horizon geometry of non-extreme black holes has a Rindler part, we note that it is plausible to find a dual CCFT at the horizon of non-extreme black holes. By using our energy-momentum tensor, we find the correct mass of non-rotating BTZ and show that the Cardy-like formula for CCFT yields the Bekenstein-Hawking entropy of non-extreme BTZ. Our current work is the first step towards describing the entropy of non-extreme black holes in terms of CCFTs microstates which live on the horizon.

KEYwords: Gauge-gravity correspondence, AdS-CFT Correspondence, Black Holes

ARXIV EPRINT: 1404.3937

Open Access, (C) The Authors.

Article funded by $\mathrm{SCOAP}^{3}$.

doi:10.1007/JHEP06(2014)134 


\section{Contents}

1 Introduction 1

2 Rindler-AdS spacetime $\quad 3$

3 From Rindler-AdS/CFT to Rindler/CCFT 4

3.1 Two-point functions of scalar operators 4

$\begin{array}{ll}3.2 & \text { A stress tensor for Rindler spacetime }\end{array}$

4 Non-extreme black holes/CCFT correspondence $\quad 8$

4.1 Entropy of non-extreme BTZ as degeneracy of CCFT states 9

5 Conclusion $\quad 11$

A Representation of CFT generators dual to the Rindler-AdS 11

\section{Introduction}

Recently, there has been a growing interest in studying holography for asymptotically flat spacetimes. One particular method for studying flat-space holography was introduced in [1-3] in which one starts from the known results of the AdS/CFT correspondence and takes their flat limit. In addition to this, various aspects of the flat-space holography were also addressed in [4-14] by merely taking flat-space limit of the AdS counterparts.

It is a well-known fact that taking the flat-space limit of calculations performed in asymptotically AdS spacetimes leads to results related to the asymptotically flat spacetimes. The flat limit of AdS is achieved by taking its large radius limit, which is equivalent to the zero cosmological constant limit. At the level of action and all covariantly constant quantities, the flat limit of AdS clearly generates the results found in the flat case. However, for the metric of asymptotically AdS spacetimes, the large radius limit must be taken carefully. ${ }^{1}$

A related question in this regard is the interpretation of the flat limit from the point of view of the boundary theory. It was argued in [1-3] that the flat limit of asymptotically AdS spacetimes corresponds to the contraction of coordinates of the boundary CFT. Accordingly, asymptotically flat spacetimes have a dual description in terms of a contracted conformal field theory (CCFT). For developing the Flat/CCFT correspondence one may start from AdS/CFT and take the appropriate limit, i.e., the large radius limit of AdS on the bulk side and contraction of coordinates on the boundary. As we will discuss in section

\footnotetext{
${ }^{1}$ An example of coordinate systems for asymptotically AdS spacetimes for which the flat limit is welldefined is given in the paper [15].
} 
3, the structure of conformal boundary dictates the coordinate which must be contracted in the CFT.

As another check for the Flat/CCFT correspondence, one can take the flat limit of the Rindler-AdS/CFT correspondence. Observers with constant acceleration in the AdS spacetimes perceive a temperature. Moreover, they do not have access to the full AdS spacetime and are restricted to the two wedge-like regions which are characterized by cosmological horizons. The coordinate system in which these observers are at rest is called Rindler-AdS coordinates whose flat limit is the Rindler spacetime. It was argued in [16] that the physics inside the two wedges of Rindler-AdS has a holographic description as entangled states of a pair of CFTs which live on the boundary of Rindler-AdS wedges. The reduced density matrix of one of these CFTs describes the spacetime inside one of the wedges up to the horizon which should be replaced by some kind of singularity. Using this holographic picture, the thermodynamics of Rindler-AdS has been recovered in [17]. The temperature and entropy of Rindler-AdS are given by using two-point function and degeneracy of states of the dual CFT.

One can use the results of Rindler-AdS/CFT correspondence in order to develop a holographic dual theory for the Rindler spacetimes. Since the flat limit of Rindler-AdS is Rindler spacetime, it is possible to use the dictionary of Flat/CCFT correspondence and find the CCFT dual to the Rindler. Similar to CFTs, one can calculate n-point functions of CCFTs (see for example $[18,19]$ ). In this paper, we perform the same method for the two-dimensional CCFT resulting from the contraction of the CFT dual to the three-dimensional Rindler-AdS and show that the two point function of scalar operators is completely consistent with the bulk calculations. Moreover, one can use the proposal of [11] for the calculation of the stress tensor of asymptotically flat gravity. We repeat the same procedure for the three-dimensional Rindler spacetimes and find non-zero components of the stress tensor which can be used in the calculation of conserved charges by using Brown and York's method [20].

Furthermore, we note that two-dimensional Rindler spacetimes appear in the near horizon geometry of non-extreme black holes. This is a property of all non-extreme black holes with any asymptotic behaviour and in any dimension. Here, we use the Rindler/CCFT correspondence and propose a dual theory for the non-extreme black holes which has near horizon information. In this view, Rindler/CCFT for non-extreme black holes is reminiscent of kerr/CFT correspondence [21] for the extremal cases. For non-rotating BTZs we check this proposal. At the first step we find the mass of non-rotating BTZ by using our proposed stress tensor for the Rindler gravity. Finally, we find the entropy of non-rotating BTZ by using Cardy-like formula of CCFTs which results in the Bekenstein-Hawking entropy. This formula has been derived in [4] and used previously for the calculation of entropy of three-dimensional flat cosmological solutions [22].

The paper is organized as follows. In section 2 we briefly review Rindler-AdS. In section 3 we find the CCFT dual to the three-dimensional Rindler spacetime and calculate two point functions of scalar operators and also energy momentum tensor of the Rindler space. Section 4 is devoted to the non-extreme/CCFT correspondence for which we use our proposal for finding a dual theory at the horizon of non-extreme BTZ. In section 5 we conclude with some future remarks. 


\section{Rindler-AdS spacetime}

An observer at $r=r_{0}$ of three-dimensional Rindler-AdS metric,

$$
d s^{2}=-\alpha^{2} r^{2} d \tau^{2}+\frac{d r^{2}}{1+\frac{r^{2}}{\ell^{2}}}+\left(1+\frac{r^{2}}{\ell^{2}}\right) d \chi^{2},
$$

perceives a constant acceleration $a_{(3)}^{2}=\frac{1}{r_{0}^{2}}+\frac{1}{\ell^{2}}$, where $\ell$ is AdS radius and proper time of observer is given by $\alpha r_{0} \tau$. Thus the time coordinate of the metric (2.1) is the proper time of an observer which is located at $r=r_{0}=\frac{1}{\alpha}$. The temperature which these observers measure, can be given by using Rindler temperature of higher dimensional flat embeding space [23-26]. Let $\left\{X^{0}, X^{1}, X^{2}, X^{3}\right\}$ denote the coordinates of embedding space with signature $(-,+,+,-)$. The metric $(2.1)$ is given by the following change of coordinates in the four-dimensional embedding space:

$$
\begin{aligned}
& X^{0}=r \sinh (\alpha \tau), \\
& X^{1}=r \cosh (\alpha \tau), \\
& X^{2}=\sqrt{\ell^{2}+r^{2}} \sinh \left(\frac{\chi}{\ell}\right), \\
& X^{3}=\sqrt{\ell^{2}+r^{2}} \cosh \left(\frac{\chi}{\ell}\right) .
\end{aligned}
$$

Thus hyperspace $r=r_{0}$ is given by

$$
\left(X^{1}\right)^{2}-\left(X^{0}\right)^{2}=r_{0}^{2}=\frac{1}{a_{(4)}^{2}}
$$

where $a_{(4)}$ is four-dimensional Rindler acceleration and the temperature is

$$
T=\frac{a_{(4)}}{2 \pi}=\frac{1}{2 \pi r_{0}}=\frac{\sqrt{a_{(3)}^{2}-\frac{1}{\ell^{2}}}}{2 \pi} .
$$

Thus the Rindler-AdS observer must have acceleration greater than a critical value $\frac{1}{\ell^{2}}$ in order to perceive a temperature. It is clear from (2.4) that the temperature which an observer with proper time $\tau$ measures is

$$
T_{\text {Rindler-AdS }}=\frac{\alpha}{2 \pi} .
$$

This must coincide with the periodicity of Euclidean time in the metric (2.1).

The relation between Rindler-AdS coordinate and global coordinate of $\mathrm{AdS}_{3}$ with metric

$$
d s^{2}=-\left(1+\frac{\rho^{2}}{\ell^{2}}\right) d t^{2}+\left(1+\frac{\rho^{2}}{\ell^{2}}\right)^{-1} d \rho^{2}+\rho^{2} d \phi^{2}
$$


is given by $[17]$

$$
\begin{aligned}
\rho^{2} & =r^{2}\left[\cosh ^{2}\left(\frac{\chi}{\ell}\right)+\sinh ^{2}(\alpha \tau)\right]+\ell^{2} \sinh ^{2}\left(\frac{\chi}{\ell}\right) \\
\tan \phi & =\frac{\sqrt{r^{2}+\ell^{2}} \sinh \left(\frac{\chi}{\ell}\right)}{r \cosh (\alpha \tau)} \\
\tan \left(\frac{t}{\ell}\right) & =\frac{r \sinh (\alpha \tau)}{\sqrt{r^{2}+\ell^{2}} \cosh \left(\frac{\chi}{\ell}\right)}
\end{aligned}
$$

From the last equation of (2.7), it is clear that in the limit $\tau \rightarrow \pm \infty$, we have $t \rightarrow \pm \frac{\ell \pi}{2}$. Thus Rindler-AdS covers a portion of global AdS which consists of two wedges. Any accelerating observer has access just to one of the wedges.

The flat limit $(\ell \rightarrow \infty)$ of the metric (2.1) is well-defined and results in Rindler space-time,

$$
d s^{2}=-\alpha^{2} r^{2} d \tau^{2}+d r^{2}+d \chi^{2}
$$

\section{From Rindler-AdS/CFT to Rindler/CCFT}

Let us start from the metric of Rindler-AdS (2.1) and try to find metric of its conformal boundary. The boundary is located at $r \rightarrow \infty$. For large and fixed $r$ we can write the metric of conformal boundary as

$$
d s_{C B}^{2}=\frac{r^{2}}{G^{2}}\left(-\alpha^{2} G^{2} d \tau^{2}+\frac{G^{2}}{\ell^{2}} d \chi^{2}\right)
$$

where $G$ is Newton constant. Here we intentionally used $G$ in the conformal factor to avoid its dependence on $\ell$. Thus the boundary is a plane with the metric $d s^{2}=-d T^{2}+d X^{2}$, where $T=\alpha G \tau$ and $X=\chi G / \ell$. From this identification it is clear that the flat limit on the bulk side, i.e. $G / \ell \rightarrow 0$, corresponds to the contraction of $X$ coordinate, i.e. $X \rightarrow \epsilon X$. Let us call the two-dimensional CFT for which the $X$ coordinate has been contracted, a contracted-CFT, or CCFT. It is argued in the rest of this paper that holographic dual of one of the Rindler wedges is a CCFT.

The fact that in the Rindler/CCFT correspondence $X$ coordinate must be contracted, is not in contrast with the original proposal of [3] for the Flat/CCFT correspondence, where time might be contracted. In fact the CFT of [3] lives on the cylinder while in the present case it is on the plane. In mapping from a cylinder to the plane, $X$ and $T$ change their roles. A way for seeing this fact is using line elements (2.1) and (2.6). It is easy to see that by making the changes $\alpha \tau \rightarrow i \phi, r \rightarrow \rho$ and $\chi \rightarrow i t$, the metric of Rindler-AdS transforms to the global coordinate. These transformations are also valid for the asymptotic Killing vectors and the generators of dual CFTs. Thus the contraction of time in the global case changes to the contraction of space in the Rindler case.

\subsection{Two-point functions of scalar operators}

For developing Rindler/CCFT, similar to Flat/CCFT correspondence, we begin with Rindler-AdS/CFT calculations and take the appropriate limit. One of the results of [17] 
is that the holographic calculation for the two-point function of two scalar operators with conformal weights $h_{1}=h_{2}=\bar{h}_{1}=\bar{h}_{2}$, located in one of the entangled CFTs, results in

$$
\left\langle\mathcal{O}\left(\chi_{1}, \tau_{1}\right) \mathcal{O}\left(\chi_{2}, \tau_{2}\right)\right\rangle=\frac{1}{\left[\cosh \left(\frac{\chi_{1}-\chi_{2}}{\ell}\right)-\cosh \left(\alpha\left(\tau_{1}-\tau_{2}\right)\right)\right]^{\Delta}},
$$

where

$$
\Delta=h+\bar{h}=1+\sqrt{1+m^{2} \ell^{2}},
$$

and $m$ is the mass of corresponding scalar filed on the bulk side.

The $\ell \rightarrow \infty$ limit (precisely $G / \ell \rightarrow 0$ limit ) of the two-point function (3.2) results in a non-zero value just for the massless case $m=0$ :

$$
\left\langle\mathcal{O}\left(\chi_{1}, \tau_{1}\right) \mathcal{O}\left(\chi_{2}, \tau_{2}\right)\right\rangle_{\text {rindler }}=\frac{1}{\left[1-\cosh \left(\alpha\left(\tau_{1}-\tau_{2}\right)\right)\right]^{\Delta_{0}}},
$$

where $\Delta_{0}=2$.

Now we want to show that symmetry considerations lead to a similar result as (3.4) for a CCFT. In order to find two point functions of scalar operators in the $X$-contracted CFT or CCFT, we should find the symmetry generators of CCFT in the first step. A way to do this calculation is to start from the symmetry generators of CFT and contract them in the manner that Flat/CCFT correspondence proposes.

The Virasoro generators of the CFT dual to the Rindler-AdS spacetime are given by

$$
\mathcal{L}_{n}=e^{-n z} \partial_{z}, \quad \overline{\mathcal{L}}_{n}=e^{-n \bar{z}} \partial_{\bar{z}}
$$

where $z=x+t$ and $\bar{z}=x-t$. In the appendix we show how this representation for the conformal symmetry is derived. ${ }^{2}$

According to the dictionary of Flat/CCFT correspondence [1-3], the generators of contracted conformal algebra are given by

$$
L_{n}=\mathcal{L}_{n}-\overline{\mathcal{L}}_{-n}, \quad M_{n}=\epsilon\left(\mathcal{L}_{n}+\overline{\mathcal{L}}_{-n}\right),
$$

and contracting $x \rightarrow \epsilon x$. At the $\epsilon \rightarrow 0$ limit we have

$$
L_{n}=e^{-n t}\left(-n x \partial_{x}+\partial_{t}\right), \quad M_{n}=e^{-n t} \partial_{x}
$$

which satisfy:

$$
\left[L_{m}, L_{n}\right]=(m-n) L_{m+n}, \quad\left[L_{m}, M_{n}\right]=(m-n) M_{m+n}, \quad\left[M_{m}, M_{n}\right]=0 .
$$

This algebra is isomorphic to the $\mathrm{BMS}_{3}$ algebra [27, 28] as the asymptotic symmetry of three dimensional asymptotically flat spacetimes.

Since the flat limit in the bulk calculation of correlators is non-zero for the massless case with a finite value of scaling dimension $\Delta=\Delta_{0}=2$, we restrict ourselves to this particular case. In order to find two-point function of the scalar operator, we need $\delta_{\tilde{\xi}} \mathcal{O}$

\footnotetext{
${ }^{2}$ The dimensionless coordinates $x$ and $t$ are related to the $\chi$ and $\tau$ coordinates of the bulk geometry by $t=\alpha \tau$ and $x=\chi / \ell$.
} 
which is the variation of the operator $\mathcal{O}$ under the symmetry transformation $\tilde{\xi}$. We want to find this quantity for the CCFT by using the analogue transformation in the CFT which for our case is given by

$$
\delta_{\xi} \mathcal{O}=\frac{\Delta_{0}}{2}\left(\partial_{z} \xi^{z}+\partial_{\bar{z}} \xi^{\bar{z}}\right) \mathcal{O}+\left(\xi^{z} \partial_{z}+\xi^{\bar{z}} \partial_{\bar{z}}\right) \mathcal{O} .
$$

Using the following identities between CCFT and CFT elements,

$$
\begin{array}{rlrl}
\tilde{z}+\overline{\tilde{z}} & =\lim _{\epsilon \rightarrow 0} \frac{1}{\epsilon}(z+\bar{z}), & \tilde{z}-\overline{\tilde{z}}=\lim _{\epsilon \rightarrow 0}(z-\bar{z}), \\
\xi^{\tilde{z}}+\xi^{\bar{z}}=\lim _{\epsilon \rightarrow 0} \frac{1}{\epsilon}\left(\xi^{z}+\xi^{\bar{z}}\right), & \xi^{\tilde{z}}-\xi^{\overline{\tilde{z}}}=\lim _{\epsilon \rightarrow 0}\left(\xi^{z}-\xi^{\bar{z}}\right) \\
\partial_{\tilde{z}}+\partial_{\tilde{\tilde{z}}}=\lim _{\epsilon \rightarrow 0} \epsilon\left(\partial_{z}+\partial_{\bar{z}}\right), & \partial_{\tilde{z}}-\partial_{\tilde{z}}=\lim _{\epsilon \rightarrow 0}\left(\partial_{z}-\partial_{\bar{z}}\right),
\end{array}
$$

where tilde shows the corresponding elements in the CCFT, one can check that

$$
\delta_{\tilde{\xi}} \mathcal{O}=\lim _{\epsilon \rightarrow 0} \delta_{\xi} \mathcal{O}=\frac{\Delta_{0}}{2}\left(\partial_{\tilde{z}} \xi^{\tilde{z}}+\partial_{\overline{\tilde{z}}} \xi^{\overline{\tilde{z}}}\right) \mathcal{O}+\left(\xi^{\tilde{z}} \partial_{\tilde{z}}+\xi^{\bar{z}} \partial_{\bar{z}}\right) \mathcal{O} .
$$

Lets denote the two-point function of scalar operator in the CCFT by $G_{2}\left(t_{1}, x_{1} ; t_{2}, x_{2}\right)=\left\langle\mathcal{O}\left(t_{1}, x_{1}\right) \mathcal{O}\left(t_{2}, x_{2}\right)\right\rangle$. This must be invariant under the $M_{n}$ and $L_{n}$ for $n=0, \pm 1$. Using (3.7) and (3.13), we find that

$$
\begin{aligned}
\left(e^{-n t_{1}} \partial_{x_{1}}+e^{-n t_{2}} \partial_{x_{2}}\right) G_{2}\left(t_{1}, x_{1} ; t_{2}, x_{2}\right) & =0 \\
{\left[-n \Delta_{0}\left(e^{-n t_{1}}+e^{-n t_{2}}\right)+e^{-n t_{1}}\left(\partial_{t_{1}}-n x_{1} \partial_{x_{1}}\right)\right.} & \\
\left.+e^{-n t_{2}}\left(\partial_{t_{2}}-n x_{2} \partial_{x_{2}}\right)\right] G_{2}\left(t_{1}, x_{1} ; t_{2}, x_{2}\right) & =0 .
\end{aligned}
$$

These equations determine the two-point function as

$$
G_{2}\left(t_{1}, x_{1} ; t_{2}, x_{2}\right)=\frac{C}{\left(\sinh \frac{t_{1}-t_{2}}{2}\right)^{2 \Delta_{0}}},
$$

which is the same as (3.4).

\subsection{A stress tensor for Rindler spacetime}

Using Rindler/CCFT correspondence, we can define a stress tensor for the Rindler spacetime. The connection is similar to the generic rule of AdS/CFT, i.e., the stress tensor of gravity is given by one pint function of energy-momentum tensor of dual theory. Since in developing Flat/CCFT correspondence we find all quantities by the flat limit of AdS/CFT calculations, we apply the same procedure for the stress tensor as well. In fact the method of calculation of the flat-space energy-momentum tensor has been introduced earlier in the paper [11]. ${ }^{3}$ The stress tensor of flat gravity is given by one point functions of the contracted CFT resulting from contracting the one point functions of the original CFT.

\footnotetext{
${ }^{3}$ The holographic renormalization approach for the flat space holography has been also studied in [29] and [30], but non of them addressed the connection to contracted CFT.
} 
In this section we use the same method for finding the stress tensor of three-dimensional Rindler spacetimes. The starting point is Rindler-AdS metric (2.1) and the definition of Brown and York's quasi-local energy momentum tensor [20],

$$
T^{\mu \nu}=\frac{2}{\sqrt{-\gamma}} \frac{\delta S}{\delta \gamma_{\mu \nu}}
$$

where $S=S_{\text {grav }}\left(\gamma_{\mu \nu}\right)$ is the gravitational action viewed as a functional of boundary metric $\gamma_{\mu \nu}$,

$$
S=\frac{1}{16 \pi G} \int_{\mathcal{M}} d^{3} x \sqrt{-g}\left(R-\frac{2}{\ell^{2}}\right)-\frac{1}{8 \pi G} \int_{\partial \mathcal{M}} d^{2} x \sqrt{-\gamma} \mathcal{K}-\frac{1}{8 \pi G \ell} \int_{\partial \mathcal{M}} d^{2} x \sqrt{-\gamma}
$$

The second term is Gibbons-Hawking term and the last one is the counterterm action that must be added in order to obtain a finite stress tensor, and $\mathcal{K}$ is trace of the extrinsic curvature of the boundary

$$
\mathcal{K}=\gamma^{\mu \nu} \mathcal{K}_{\mu \nu}=\gamma^{\mu \nu} \gamma_{\mu}^{\rho} \nabla_{\rho} n_{\nu}
$$

where $\gamma_{\mu \nu}=g_{\mu \nu}-n_{\mu} n_{\nu}$ and $n_{\nu}$ is the outward pointing normal vector to the boundary $\partial \mathcal{M}$. (3.17) and (3.18) result in

$$
T_{\mu \nu}=-\frac{1}{8 \pi G}\left(\mathcal{K}_{\mu \nu}-\mathcal{K} \gamma_{\mu \nu}+\frac{\gamma_{\mu \nu}}{\ell}\right)
$$

At the boundary of Rindler-AdS (2.1) i.e. $r \rightarrow \infty$, the non-zero components of $T_{\mu \nu}$ are

$$
T_{\tau \tau}=\frac{\alpha^{2} \ell}{16 \pi G}, \quad T_{\chi \chi}=\frac{1}{16 \pi G \ell} .
$$

It is clear that the flat limit $G / \ell \rightarrow 0$ is not well-defined for (3.21). In order to define stress tensor of Rindler spacetime,$\tilde{T}_{\mu \nu}$, by taking the flat limit of the stress tensor of Rindler-AdS ,$T_{\mu \nu}$, we use the proposal of [11] and write

$$
\begin{aligned}
& \tilde{T}_{++}+\tilde{T}_{--}=\lim _{\frac{G}{\ell} \rightarrow 0}\left[\frac{G}{\ell}\left(T_{++}+T_{--}\right)\right], \\
& \tilde{T}_{++}-\tilde{T}_{--}=\lim _{\frac{G}{\ell} \rightarrow 0}\left[T_{++}-T_{--}\right],
\end{aligned}
$$

where the light-cone coordinates in the Rindler-AdS are given by $x^{ \pm}=\frac{G}{\ell} \chi \pm \alpha G \tau$, while in Rindler spacetime we define the light-cone coordinates as $\tilde{x}^{ \pm}=\chi \pm \alpha G \tau$ with $\tau$ and $\chi$ being coordinates of (2.8). The $\tilde{T}_{+-}$component like $T_{+-}$is assumed to be zero. The reader can easily check that with the definition $(3.22), \tilde{T}_{++}$and $\tilde{T}_{--}$are both finite, and finally for the Rindler spacetime we have

$$
\tilde{T}_{\tau \tau}=\frac{\alpha^{2}}{16 \pi}, \quad \tilde{T}_{\chi \chi}=\frac{1}{16 \pi G^{2}} .
$$

Since the dual CCFT of Rindler is constructed by contracting space-like coordinate i.e. ,$x \rightarrow \epsilon x$, it is natural to expect that its momentum operator is found from the original 
CFT by a similar scaling. The first line of (3.22) is the corresponding equation for the scaling of momentum from the bulk point of view. The second equation of (3.22) shows that Hamiltonian of CCFT is the same as that of the original CFT. This is justified by the fact that our contraction does not affect the time coordinate.

The point which we used implicitly in the definition (3.22) of the stress tensor for Rindler spacetime (but will be explicitly used in the rest of this section) is that we assume that similar to the original CFT, CCFT lives on a plane. The metric of this plane is similar to the CFT case but with the difference that its $x$ coordinate is the contracted one. This fact is clear in the definition of the light-cone coordinates $x^{ \pm}$and $\tilde{x}^{ \pm}$for Rindler-AdS and Rindler spacetimes, respectively, where the limit $G / \ell \rightarrow 0$ in the bulk corresponds to the limit $\epsilon \rightarrow 0$ on the boundary. In summary, the CCFT dual to the Rindler spacetime

$$
d s^{2}=-\alpha^{2} r^{2} d \tau^{2}+d r^{2}+d \chi^{2}
$$

lives on a plane with metric

$$
d s_{\partial_{M}}^{2}=-\alpha^{2} G^{2} d \tau^{2}+d \chi^{2}
$$

\section{Non-extreme black holes/CCFT correspondence}

The energy momentum of previous section for Rindler spacetime can be used in the computation of conserved charges of non-extreme BTZ. If one takes the near horizon limit of non-extreme black holes with any asymptotic behaviour, the resulting geometry has a Rindler part. Let us study the simplest case which is non-rotating BTZ with the metric

$$
d s^{2}=-f(\rho) d \tau^{2}+f(\rho)^{-1} d \rho^{2}+\rho^{2} d \phi^{2},
$$

where $f(\rho)=\frac{\rho^{2}}{\ell^{2}}-8 G M$ and $M$ is the mass of the black hole. If we denote the outer horizon by $\rho_{h}$, which is given by $\rho_{h}=\ell \sqrt{8 G M}$, and define a new coordinate $y=\rho-\rho_{h}$, in the near horizon characterized by $y \ll \rho_{h}$, we find

$$
d s_{N H}^{2}=-f^{\prime}\left(\rho_{h}\right) y d \tau^{2}+\left(f^{\prime}\left(\rho_{h}\right) y\right)^{-1} d y^{2}+\rho_{h}^{2} d \phi^{2} .
$$

Defining a new coordinate $r$ by $d r=d y / \sqrt{f^{\prime}\left(\rho_{h}\right) y}$ results in

$$
d s_{N H}^{2}=-\frac{f^{\prime 2}\left(\rho_{h}\right)}{4} r^{2} d \tau^{2}+d r^{2}+\rho_{h}^{2} d \phi^{2} .
$$

Comparing (4.3) with (3.24) shows that the near horizon metric is Rindler with $\alpha=$ $f^{\prime}\left(\rho_{h}\right) / 2=\sqrt{8 G M} / \ell$ and a compact $\chi$ coordinate, given by $\chi=\rho_{h} \phi .{ }^{4}$

Using (3.23), we find the non-zero component of the stress tensor as

$$
\tilde{T}_{\tau \tau}=\frac{\alpha^{2}}{16 \pi}=\frac{G M}{2 \pi \ell^{2}}, \quad \tilde{T}_{\phi \phi}=\frac{\rho_{h}^{2}}{16 \pi G^{2}}=\frac{\ell^{2} M}{2 \pi G} .
$$

\footnotetext{
${ }^{4} \ell$ in the parameter $\alpha$ and also $\rho_{h}$ is not radius of AdS in the metric of Rindler-AdS. Thus taking the limit $\ell \rightarrow \infty$ from the calculations of Rindler-AdS does not affect $\alpha$ and $\rho_{h}$.
} 
Let us use (4.4) and compute the conserved charge of killing vector $\xi=\partial_{\tau}$. If we use the metric

$$
d s_{\partial_{M}}^{2}=-\alpha^{2} G^{2} d \tau^{2}+\rho_{h}^{2} d \phi^{2},
$$

in the Brown and York's formula

$$
Q_{\xi}=\int_{\Sigma} d \phi \sqrt{\sigma} v^{\mu} \xi^{\nu} \tilde{T}_{\mu \nu}
$$

where $\Sigma$ is the spacelike surface of $\partial M$ ( $\tau=$ constant surface), $\sigma_{a b}$ is metric of $\Sigma$ i.e. $\sigma_{a b} d x^{a} d x^{b}=\rho_{h}^{2} d \phi^{2}$ and $v^{\mu}$ is the unit time-like vector normal to $\Sigma$, we find

$$
Q_{\partial_{\tau}}=\frac{\alpha \rho_{h}}{8 G}=M
$$

Thus our stress tensor results in the mass of the non-rotating BTZ as the charge of killing vector $\xi=\partial_{\tau}$, where $\tau$ is dimension-full time of near horizon geometry. This is a non-trivial check for our proposed energy-momentum tensor of Rindler spacetime.

\subsection{Entropy of non-extreme BTZ as degeneracy of CCFT states}

Our discussion so far, proposes a dual two-dimensional CCFT for the three-dimensional Rindler spacetime. Moreover, since the near horizon geometry of non-extreme black holes has a Rindler part, it is plausible to propose a dual description for the non-extreme black holes at the horizon. This dual theory is a CCFT, which has contracted conformal symmetry. In two-dimensions this symmetry is given by (3.8). If we start from two copies of conformal algebra with central charges $c$ and $\bar{c}$ and define generators of CCFT as (3.6), the $\left[L_{n}, L_{m}\right]$ and $\left[L_{n}, M_{m}\right]$ commutators will have central extensions. Let us denote the central charge of $\left[L_{n}, L_{m}\right]$ by $c_{L}$ and central charge of $\left[L_{n}, M_{m}\right]$ by $c_{M}$. They are given by taking the following limit of the central charges of the original CFT:

$$
\begin{aligned}
c_{L} & =\lim _{\epsilon \rightarrow 0} \frac{1}{12}(c-\bar{c}), \\
c_{M} & =\lim _{\epsilon \rightarrow 0} \frac{\epsilon}{12}(c+\bar{c}) .
\end{aligned}
$$

According to the Flat/CCFT proposal, the $\epsilon \rightarrow 0$ limit on the boundary theory corresponds to $G / \ell \rightarrow 0$ in the bulk. If we use Brown and Henneaux's central charges $c=\bar{c}=3 \ell / 2 G[31]$, the central charge $c_{L}$ will be zero but $c_{M}=1 / 4$.

The degeneracy of states $\left|h_{L}, h_{M}\right\rangle$ which are eigenstates of $L_{0}$ and $M_{0}$ with eigenvalues $h_{L}$ and $h_{M}$, can be given by a Cardy like formula for the CCFT. This formula has been given in [4] for large charges as

$$
S=\ln d\left(h_{L}, h_{M}\right)=2 \pi\left(c_{L} \sqrt{\frac{h_{M}}{2 c_{M}}}+h_{L} \sqrt{\frac{c_{M}}{2 h_{M}}}\right)
$$

where $\left(h_{L}, h_{M}\right)$ are given in terms of conformal weights $(h, \bar{h})$ of the original CFT as

$$
h_{L}=\lim _{\epsilon \rightarrow 0}(h-\bar{h}), \quad h_{M}=\lim _{\epsilon \rightarrow 0} \epsilon(h+\bar{h}),
$$


If our proposal for the dual CCFT at the horizon of non-extreme black holes is correct, the entropy of non-extreme BTZ must be given by such a formula in terms of degeneracy of states of CCFT. In the following we will show this fact for the entropy of non-rotating BTZ.

The first step is the calculation of $h_{M}$ and $h_{L}$. They are given by (4.10) from conformal weights $(h, \bar{h})$ of original CFT. The original CFT is dual to Rindler-AdS spacetimes for which the flat limit results in near horizon geometry of BTZ. Thus the metric of RindlerAdS is given by (2.1) but its $\chi$ coordinate should be compactified as $\chi=\rho_{h} \phi$, where $\rho_{h}$ is radius of horizon of BTZ and it is just a parameter in this stage; the $\phi$ coordinate takes values in the interval $[0,2 \pi]$. Now the entropy of the cosmological horizon of Rindler-AdS located at $r=0$ is finite and is given by $S=\pi \rho_{h} / 2 G$. Using the Rindler-AdS/CFT correspondence, this entropy must be given by Cardy formula in terms of degeneracy of states of CFT. Let us write Cardy formula in the following form

$$
S=\frac{\pi^{2}}{3}\left(c T_{R}+\bar{c} T_{L}\right)
$$

where right and left temperatures of CFT are given by

$$
\frac{1}{T_{R}}=\pi \sqrt{\frac{c}{6 h}}, \quad \frac{1}{T_{L}}=\pi \sqrt{\frac{\bar{c}}{6 \bar{h}}} .
$$

Moreover, $T_{L}$ and $T_{R}$ are related to the temperature $T=\alpha / 2 \pi$ of Rindler-AdS as

$$
\frac{1}{T}=\frac{\ell}{2}\left(\frac{1}{T_{R}}+\frac{1}{T_{L}}\right)
$$

Thus we can calculate $h$ and $\bar{h}$. The final result is

$$
\begin{aligned}
& h=\frac{\rho_{h}^{2}}{16 \ell G}\left(2-\frac{\alpha \ell^{2}}{\rho_{h}}+2 \sqrt{1-\frac{\alpha \ell^{2}}{\rho_{h}}}\right), \\
& \bar{h}=\frac{\rho_{h}^{2}}{16 \ell G}\left(2-\frac{\alpha \ell^{2}}{\rho_{h}}-2 \sqrt{1-\frac{\alpha \ell^{2}}{\rho_{h}}}\right)
\end{aligned}
$$

Now we can calculate $\left(h_{L}, h_{M}\right)$ of CCFT dual to Rindler spacetime. They are given by (4.10) where $\epsilon$ must be identified with $G / \ell$. Using (4.14), the $G / \ell \rightarrow 0$ is well-defined and results in:

$$
h_{L}=\frac{\rho_{h}^{2}}{4 G} \sqrt{-\frac{\alpha}{\rho_{h}}}, \quad h_{M}=-\frac{\alpha \rho_{h}}{8} .
$$

Putting everything in the Cardy-like formula (4.9) gives

$$
S=\frac{\pi \rho_{h}}{2 G}
$$

which is exactly the Bekenstein-Hawking entropy of non-extremal BTZ. 


\section{Conclusion}

Our calculations in this paper uncover the existence of a two-dimensional dual CCFT for gravity in three-dimensional Rindler spacetimes. There are several points related to this duality which we did not address in this paper. The hypersurface on which CCFT lives, i.e., the metric (3.25) is constructed by an anisotropic scaling of Rindler space. It seems that this kind of anisotropic scaling is necessary for studying flat-space holography. Moreover, for the current case of Rindler holography, the dual theory is at spacial infinity rather than Null infinity of flat spacetimes. This point does not in contrast with previously known results about flat-space holography but needs more study. ${ }^{5}$

The independence of correlation function (3.16) from space coordinates is another interesting property of Rindler-space holography. These correlators may reveal some properties of CCFTs dual to asymptotically flat spacetimes.

The non-extreme black holes/CCFT correspondence which we propose in this paper is the first step in this direction and needs careful studies in higher dimensions. Since there is no decoupling between near-horizon region and rest of space for the non-extreme black holes, the information which the horizon CCFT may provide is problematic. The main question is how much information this dual theory has about the inside region of horizon, its hairs and entropy of black hole. Another point which relations (4.15) reveal, is the unusual values of $h_{L}$ and $h_{M}$. One of them is negative and another is imaginary, but in the Cardy-like formula they compensate each other and the final entropy is well-defined. The relation between CCFTs and the proposals of $[33,34]$ for the dual theory of horizons which has a chiral Virasoro symmetry is another interesting question in this regard. ${ }^{6}$

All the above points are signs of non-triviality of CCFTs dual to flat spacetimes and may uncover new facts about gravity in the flat spacetimes. We hope to address the above points in our future works.

\section{Acknowledgments}

The authors would like to thank M. Alishahiha, D. Allahbakhshi, A. Bagchi, D. Grumiller, A. Hosseiny, A. E. Mosaffa, M. Safari and J. Simon for useful comments and discussions. We also especially thank D. Kaviani for his comments on the revised version.

\section{A Representation of CFT generators dual to the Rindler-AdS}

Symmetry generators can be given by starting from Killing vectors of Rindler-AdS (2.1), restricting them to the $(\tau, \chi)$ components and taking $r \rightarrow \infty$ limit. This could result in the global part of conformal algebra and one can extend them to find full symmetry. Using generators of 4 dimensional flat embedding space, we can find Killing vectors of

\footnotetext{
${ }^{5}$ The BMS symmetry also exist at the spacial infinity of asymptotically flat spacetimes [32].

${ }^{6} \mathrm{~A}$ comment on the relation of this chiral Virasoro and equipartition of energy is given in section 4.3 of review [35].
} 
Rindler-AdS as

$$
\begin{aligned}
& J_{01}=-\frac{1}{\alpha} \partial_{\tau} \\
& J_{02}=-\frac{r \ell}{\sqrt{r^{2}+\ell^{2}}} \sinh (\alpha \tau) \cosh \left(\frac{\chi}{\ell}\right) \partial_{\chi} \\
& -\sqrt{r^{2}+\ell^{2}} \sinh \left(\frac{\chi}{\ell}\right)\left[\frac{\cosh (\alpha \tau)}{\alpha r} \partial_{\tau}-\sinh (\alpha \tau) \partial_{r}\right] \\
& J_{03}=\frac{r \ell}{\sqrt{r^{2}+\ell^{2}}} \sinh (\alpha \tau) \sinh \left(\frac{\chi}{\ell}\right) \partial_{\chi} \\
& +\sqrt{r^{2}+\ell^{2}} \cosh \left(\frac{\chi}{\ell}\right)\left[\frac{\cosh (\alpha \tau)}{\alpha r} \partial_{\tau}-\sinh (\alpha \tau) \partial_{r}\right] \\
& J_{12}=-\frac{r \ell}{\sqrt{r^{2}+\ell^{2}}} \cosh (\alpha \tau) \cosh \left(\frac{\chi}{\ell}\right) \partial_{\chi} \\
& +\sqrt{r^{2}+\ell^{2}} \sinh \left(\frac{\chi}{\ell}\right)\left[\frac{\sinh (\alpha \tau)}{\alpha r} \partial_{\tau}-\cosh (\alpha \tau) \partial_{r}\right] \\
& J_{13}=-\frac{r \ell}{\sqrt{r^{2}+\ell^{2}}} \cosh (\alpha \tau) \sinh \left(\frac{\chi}{\ell}\right) \partial_{\chi} \\
& -\sqrt{r^{2}+\ell^{2}} \cosh \left(\frac{\chi}{\ell}\right)\left[\frac{\sinh (\alpha \tau)}{\alpha r} \partial_{\tau}-\cosh (\alpha \tau) \partial_{r}\right] \\
& J_{23}=\ell \partial_{\chi}
\end{aligned}
$$

Taking $r \rightarrow \infty$ and defining $t=\alpha \tau$ and $x=\chi / \ell$ and restricting to the $x$ and $t$ components gives the generators of global part of conformal algebra as

$$
\begin{aligned}
& j_{01}=-\partial_{t} \\
& j_{02}=-\sinh t \cosh x \partial_{x}-\sinh x \cosh t \partial_{t} \\
& j_{03}=\sinh t \sinh x \partial_{x}+\cosh x \cosh t \partial_{t} \\
& j_{12}=\cosh t \cosh x \partial_{x}+\sinh x \sinh t \partial_{t} \\
& j_{13}=-\cosh t \sinh x \partial_{x}-\cosh x \sinh t \partial_{t} \\
& j_{23}=\partial_{x}
\end{aligned}
$$

The $\mathrm{SL}(2, R) \times \mathrm{SL}(2, R)$ generators are given by

$$
\begin{aligned}
& \mathcal{L}_{0}=\frac{1}{2}\left(j_{23}-j_{01}\right), \quad \mathcal{L}_{+1}=\frac{1}{2}\left(j_{02}+j_{03}+j_{12}+j_{13}\right), \quad \mathcal{L}_{-1}=-\frac{1}{2}\left(j_{02}-j_{03}-j_{12}+j_{13}\right) \\
& \overline{\mathcal{L}}_{0}=\frac{1}{2}\left(j_{23}+j_{01}\right), \quad \overline{\mathcal{L}}_{-1}=\frac{1}{2}\left(j_{02}-j_{03}+j_{12}-j_{13}\right), \quad \overline{\mathcal{L}}_{1}=-\frac{1}{2}\left(j_{02}+j_{03}-j_{12}-j_{13}\right)
\end{aligned}
$$

which can be written as

$$
\mathcal{L}_{n}=e^{-n z} \partial_{z}, \quad \overline{\mathcal{L}}_{n}=e^{-n \bar{z}} \partial_{\bar{z}}
$$

where $z=x+t$ and $\bar{z}=x-t$. 
Open Access. This article is distributed under the terms of the Creative Commons Attribution License (CC-BY 4.0), which permits any use, distribution and reproduction in any medium, provided the original author(s) and source are credited.

\section{References}

[1] A. Bagchi, Correspondence between asymptotically flat spacetimes and nonrelativistic conformal field theories, Phys. Rev. Lett. 105 (2010) 171601 [INSPIRE].

[2] A. Bagchi, The BMS/GCA correspondence, arXiv:1006.3354 [INSPIRE].

[3] A. Bagchi and R. Fareghbal, BMS/GCA redux: towards flatspace holography from non-relativistic symmetries, JHEP 10 (2012) 092 [arXiv: 1203.5795] [INSPIRE].

[4] A. Bagchi, S. Detournay, R. Fareghbal and J. Simon, Holography of 3D flat cosmological horizons, Phys. Rev. Lett. 110 (2013) 141302 [arXiv:1208.4372] [INSPIRE].

[5] A. Bagchi, S. Detournay and D. Grumiller, Flat-space chiral gravity, Phys. Rev. Lett. 109 (2012) 151301 [arXiv:1208.1658] [INSPIRE].

[6] A. Bagchi, S. Detournay, D. Grumiller and J. Simon, Cosmic evolution from phase transition of three-dimensional flat space, Phys. Rev. Lett. 111 (2013) 181301 [arXiv:1305.2919] [INSPIRE].

[7] A. Bagchi, Tensionless strings and galilean conformal algebra, JHEP 05 (2013) 141 [arXiv: 1303.0291] [INSPIRE].

[8] H. Afshar, A. Bagchi, R. Fareghbal, D. Grumiller and J. Rosseel, Spin-3 gravity in three-dimensional flat space, Phys. Rev. Lett. 111 (2013) 121603 [arXiv:1307.4768] [INSPIRE].

[9] H.R. Afshar, Flat/AdS boundary conditions in three dimensional conformal gravity, JHEP 10 (2013) 027 [arXiv: 1307.4855] [INSPIRE].

[10] H.A. Gonzalez, J. Matulich, M. Pino and R. Troncoso, Asymptotically flat spacetimes in three-dimensional higher spin gravity, JHEP 09 (2013) 016 [arXiv:1307.5651] [INSPIRE].

[11] R. Fareghbal and A. Naseh, Flat-space energy-momentum tensor from BMS/GCA correspondence, JHEP 03 (2014) 005 [arXiv: 1312.2109] [INSPIRE].

[12] C. Krishnan, A. Raju and S. Roy, A Grassmann path from AdS $S_{3}$ to flat space, JHEP 03 (2014) 036 [arXiv:1312.2941] [INSPIRE].

[13] A. Bagchi and R. Basu, 3D flat holography: entropy and logarithmic corrections, JHEP 03 (2014) 020 [arXiv: 1312.5748] [INSPIRE].

[14] D. Grumiller, M. Riegler and J. Rosseel, Unitarity in three-dimensional flat space higher spin theories, arXiv: 1403.5297 [INSPIRE].

[15] G. Barnich, A. Gomberoff and H.A. Gonzalez, The flat limit of three dimensional asymptotically anti-de Sitter spacetimes, Phys. Rev. D 86 (2012) 024020 [arXiv:1204.3288] [INSPIRE].

[16] B. Czech, J.L. Karczmarek, F. Nogueira and M. Van Raamsdonk, Rindler quantum gravity, Class. Quant. Grav. 29 (2012) 235025 [arXiv:1206.1323] [INSPIRE].

[17] M. Parikh and P. Samantray, Rindler-AdS/CFT, arXiv:1211.7370 [InSPIRE]. 
[18] A. Bagchi and I. Mandal, On representations and correlation functions of galilean conformal algebras, Phys. Lett. B 675 (2009) 393 [arXiv: 0903.4524] [INSPIRE].

[19] A. Akhavan, M. Alishahiha, A. Davody and A. Vahedi, Non-relativistic CFT and semi-classical strings, JHEP 03 (2009) 053 [arXiv:0811.3067] [INSPIRE].

[20] J.D. Brown and J.W. York, Quasilocal energy and conserved charges derived from the gravitational action, Phys. Rev. D 47 (1993) 1407 [gr-qc/9209012] [INSPIRE].

[21] M. Guica, T. Hartman, W. Song and A. Strominger, The Kerr/CFT correspondence, Phys. Rev. D 80 (2009) 124008 [arXiv:0809.4266] [INSPIRE].

[22] L. Cornalba and M.S. Costa, A new cosmological scenario in string theory, Phys. Rev. D 66 (2002) 066001 [hep-th/0203031] [INSPIRE].

[23] S. Deser and O. Levin, Accelerated detectors and temperature in (anti)-de Sitter spaces, Class. Quant. Grav. 14 (1997) L163 [gr-qc/9706018] [INSPIRE].

[24] S. Deser and O. Levin, Equivalence of Hawking and Unruh temperatures through flat space embeddings, Class. Quant. Grav. 15 (1998) L85 [hep-th/9806223] [INSPIRE].

[25] S. Deser and O. Levin, Mapping Hawking into Unruh thermal properties, Phys. Rev. D 59 (1999) 064004 [hep-th/9809159] [INSPIRE].

[26] T. Jacobson, Comment on 'Accelerated detectors and temperature in anti-de Sitter spaces', Class. Quant. Grav. 15 (1998) 251 [gr-qc/9709048] [INSPIRE].

[27] A. Ashtekar, J. Bicak and B.G. Schmidt, Asymptotic structure of symmetry reduced general relativity, Phys. Rev. D 55 (1997) 669 [gr-qc/9608042] [INSPIRE].

[28] G. Barnich and G. Compere, Classical central extension for asymptotic symmetries at null infinity in three spacetime dimensions, Class. Quant. Grav. 24 (2007) F15 [gr-qc/0610130] [INSPIRE].

[29] R.N. Caldeira Costa, Aspects of the zero $\Lambda$ limit in the AdS/CFT correspondence, arXiv: 1311.7339 [INSPIRE].

[30] S. Detournay, D. Grumiller, F. Scholler and J. Simon, Variational principle and 1-point functions in 3-dimensional flat space Einstein gravity, Phys. Rev. D 89 (2014) 084061 [arXiv: 1402.3687] [INSPIRE].

[31] J.D. Brown and M. Henneaux, Central charges in the canonical realization of asymptotic symmetries: an example from three-dimensional gravity,

Commun. Math. Phys. 104 (1986) 207 [INSPIRE].

[32] A. Ashtekar and R.O. Hansen, A unified treatment of null and spatial infinity in general relativity. I - Universal structure, asymptotic symmetries and conserved quantities at spatial infinity, J. Math. Phys. 19 (1978) 1542 [INSPIRE].

[33] S. Carlip, Black hole entropy from conformal field theory in any dimension, Phys. Rev. Lett. 82 (1999) 2828 [hep-th/9812013] [INSPIRE].

[34] S.N. Solodukhin, Conformal description of horizon's states, Phys. Lett. B 454 (1999) 213 [hep-th/9812056] [INSPIRE].

[35] J. Simon, Extremal black holes, holography and coarse graining, Int. J. Mod. Phys. A 26 (2011) 1903 [arXiv:1106.0116] [inSPIRE]. 